\title{
Front Matter: Volume 9370
}

, "Front Matter: Volume 9370," Proc. SPIE 9370, Quantum Sensing and Nanophotonic Devices XII, 937001 (8 February 2015); doi:

$10.1117 / 12.2185577$

SPIE. Event: SPIE OPTO, 2015, San Francisco, California, United States 


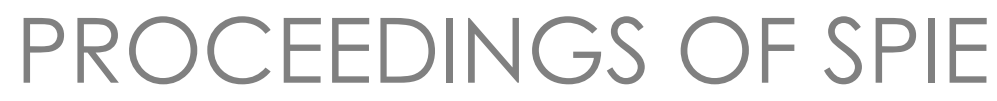

\title{
Quantum Sensing and Nanophotonic Devices XII
}

\author{
Manijeh Razeghi \\ Eric Tournié \\ Gail J. Brown \\ Editors
}

8-12 February 2015

San Francisco, California, United States

Sponsored and Published by

SPIE 
The papers included in this volume were part of the technical conference cited on the cover and title page. Papers were selected and subject to review by the editors and conference program committee. Some conference presentations may not be available for publication. The papers published in these proceedings reflect the work and thoughts of the authors and are published herein as submitted. The publisher is not responsible for the validity of the information or for any outcomes resulting from reliance thereon.

Please use the following format to cite material from this book:

Author(s), "Title of Paper," in Quantum Sensing and Nanophotonic Devices XII, edited by Manijeh Razeghi, Eric Tournié, Gail J. Brown, Proceedings of SPIE Vol. 9370 (SPIE, Bellingham, WA, 2015) Article CID Number.

ISSN: 0277-786X

ISBN: 9781628414608

\section{Published by}

\section{SPIE}

P.O. Box 10, Bellingham, Washington 98227-0010 USA

Telephone +1 3606763290 (Pacific Time) · Fax +1 3606471445

SPIE.org

Copyright @ 2015, Society of Photo-Optical Instrumentation Engineers.

Copying of material in this book for internal or personal use, or for the internal or personal use of specific clients, beyond the fair use provisions granted by the U.S. Copyright Law is authorized by SPIE subject to payment of copying fees. The Transactional Reporting Service base fee for this volume is $\$ 18.00$ per article (or portion thereof), which should be paid directly to the Copyright Clearance Center (CCC), 222 Rosewood Drive, Danvers, MA 01923. Payment may also be made electronically through CCC Online at copyright.com. Other copying for republication, resale, advertising or promotion, or any form of systematic or multiple reproduction of any material in this book is prohibited except with permission in writing from the publisher. The CCC fee code is 0277-786X/15/\$18.00.

Printed in the United States of America.

Publication of record for individual papers is online in the SPIE Digital Library.

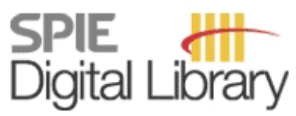

SPIEDigitalLibrary.org

Paper Numbering: Proceedings of SPIE follow an e-First publication model, with papers published first online and then in print. Papers are published as they are submitted and meet publication criteria. A unique citation identifier (CID) number is assigned to each article at the time of the first publication. Utilization of CIDs allows articles to be fully citable as soon as they are published online, and connects the same identifier to all online, print, and electronic versions of the publication. SPIE uses a six-digit CID article numbering system in which:

- The first four digits correspond to the SPIE volume number.

- The last two digits indicate publication order within the volume using a Base 36 numbering

system employing both numerals and letters. These two-number sets start with 00, 01, 02, 03, 04, 05, 06, 07, 08, 09, 0A, OB ... 0Z, followed by 10-1Z, 20-2Z, etc.

The CID Number appears on each page of the manuscript. The complete citation is used on the first page, and an abbreviated version on subsequent pages. 


\title{
Contents
}

\author{
ix Authors \\ xiii Conference Committee
}

\section{IR EMITTERS AND NEAR-FIELD CHARACTERIZATION}

937004 Metallic metasurface as a directional and monochromatic thermal emitter (Invited Paper) [9370-3]

937005 Magnetic dipole and electric dipole resonances in $\mathrm{TiO}_{2}$ microspheres at terahertz frequencies [9370-4]

937007 Characterization techniques for semiconductors and nanostructures: a review of recent advances (Invited Paper) [9370-6]

THZ SOURCES

937008 Optically pumped continuous-wave terahertz sources (Invited Paper) [9370-7]

9370 OA THz quantum cascade lasers based on a hyperuniform design (Invited Paper) [9370-9]

$9370 \mathrm{OB} \quad$ Uni-travelling carrier photodetectors as THz detectors and emitters [9370-10]

MID-IR QCL STRUCTURES

9370 OD Hydride vapour phase epitaxy assisted buried heterostructure quantum cascade lasers for sensing applications (Invited Paper) [9370-12]

9370 OE Quantum-cascade-laser active regions on metamorphic buffer layers (Invited Paper) [9370-13]

9370 OF Low power-consumption quantum cascade lasers [9370-14]

\section{IR PHOTODETECTOR MATERIALS}

$9370 \mathrm{OH} \quad$ Impact of MBE deposition conditions on InAs/GalnSb superlattices for very long wavelength infrared detection [9370-16]

9370 0J Temperature dependent carrier lifetime measurements of InAs/InAsSb T2SLs [9370-18] 
9370 OK Ideal performance of and defect-assisted carrier recombination in MWIR and LWIR InAs/InAsSb superlattice detectors [9370-19]

$9370 \mathrm{OL} \quad$ Magnetotransport potentials for anisotropic thin films with stripline and ground plane contacts (Invited Paper) [9370-20]

\section{SB-BASED IR PHOTODETECTORS}

9370 OM Room temperature performance of mid-wavelength infrared InAsSb nBn detectors [9370-21]

9370 ON MWIR InSb detector with nBn architecture for high operating temperature [9370-22]

$937000 \quad$ MTF performance: measurements, modelisation, and optimization for Sofradir II-VI IR photodetectors [9370-23]

\section{OPTOMECHANICS/MICRO-RESONATORS}

9370 OR Chaotic behaviour of photonic crystals resonators (Invited Paper) [9370-26]

9370 ОT Converting mid-infrared signals to near-infrared through optomechanical transduction [9370-28]

9370 OU Electric and magnetic dipoles in the Lorentz and Einstein-Laub formulations of classical electrodynamics (Invited Paper) [9370-29]

\section{IR SENSING}

9370 OV Quantum cascade laser-based sensor system for nitric oxide detection (Invited Paper) [9370-30]

9370 OW Quantum cascade laser-based multipass absorption system for hydrogen peroxide detection [9370-32]

9370 OX New approaches in quartz-enhanced photoacoustic sensing [9370-33]

9370 OY Quartz-enhanced photoacoustic sensors for $\mathrm{H}_{2} \mathrm{~S}$ trace gas detection [9370-34]

$9370 \mathrm{OZ}$ Use of external cavity quantum cascade laser compliance voltage in real-time trace gas sensing of multiple chemicals [9370-35]

937010 Field test results of compound specific isotope analyzer based on quantum cascade lasers and hollow waveguide [9370-36] 
QCLS AND APPLICATIONS I

937011 Quantum cascade laser stabilization at sub-Hz-level by use of a frequency comb and an optical link (Invited Paper) [9370-37]

937012 Widely tunable quantum cascade lasers for spectroscopic sensing (Invited Paper) [9370-38]

937013 Quantum cascade lasers with optical feedback: regular multimode dynamics (Invited Paper) [9370-39]

937014 Nonlinear dynamics of quantum cascade lasers with optical feedback [9370-40]

937015 Nonlinear frequency mixing in QCL-based interferometry: beyond the intrinsic resolution [9370-41]

937016 Mid-IR quantum cascade laser mode coupling in hollow-core fiber optic waveguides with single-mode beam delivery [9370-42]

\section{KEYNOTE SESSION}

937017 Selective area growth of III-nitride nanorods on polar, semi-polar, and non-polar orientations: device applications (Keynote Paper) [9370-43]

PLASMONIC DETECTION AND SENSING

937018 Enhanced two-photon-absorption using sub-wavelength antennas (Invited Paper) [9370-44]

937019 Investigation of plasmonic enhancement in a quantum dot-in-a-well structure [9370-45]

9370 IA 3D hollow nanostructures as high quality plasmonic nanocavities for multipurpose application (Invited Paper) [9370-46]

9370 1B Plasmonic characteristics of metallic nano-ring structures [9370-47]

$93701 \mathrm{C}$ Photonic and plasmonic modulators based on optical switching in $\mathrm{VO}_{2}$ (Invited Paper) [9370-48]

\section{QCLS AND APPLICATIONS II}

$93701 D$ Quantum cascade laser THz metrology (Invited Paper) [9370-49]

9370 IE Octave-spanning THz quantum cascade laser [9370-50]

$9370 \mathrm{IF} \quad$ Mid-IR and terahertz digital holography based on quantum cascade lasers [9370-51] 
$937011 \quad$ All solid state mid-infrared dual-comb spectroscopy plafform based on QCL technology (Invited Paper) [9370-54]

\section{INTEGRATION TECHNOLOGIES}

$93701 \mathrm{~J} \quad$ Mid-IR integrated photonics for sensing applications (Invited Paper) [9370-55]

$93701 \mathrm{~K} \quad$ Fabrication of silica integrated waveguide circuits for quantum enhanced sensing, quantum information processing and number resolving detection (Invited Paper) [9370-56]

$93701 \mathrm{~L} \quad$ Advances in three-dimensional integration technologies in support of infrared focal plane arrays (Invited Paper) [9370-57]

\section{RECENT ADVANCES IN QUANTUM SENSING AND NANOPHOTONICS I}

$93701 \mathrm{M}$ Polarimetric determination of the orientation of a single nano-emitter (Invited Paper) [9370-106]

9370 IP Helmholtz resonator for electric field enhancement from visible to far-infrared [9370-95]

\section{RECENT ADVANCES IN QUANTUM SENSING AND NANOPHOTONICS II}

9370 is Low noise InGaAs/InP single-photon detector for singlet oxygen detection [9370-89]

9370 1T InGaAs/InP single-photon detector with low noise, low timing jitter and high count rate [9370-90]

$93701 \mathrm{U}$ High linearity SPAD and TDC array for TCSPC and 3D ranging applications [9370-91]

$93701 \mathrm{~V} \quad$ Nitrogen vacancies (NV) centers in diamond for magnetic sensors and quantum sensing (Invited Paper) [9370-31]

\section{KEYNOTE SESSION}

$937019 \quad$ Turning assistive machines into assistive robots (Keynote Paper) [9370-58]

\section{IR PHOTODETECTORS}

$937012 \quad$ Flexibility properties of type-II InAs/GaSb SL to design MWIR pin photodiodes (Invited Paper) [9370-59]

937020 Physics and technology of antimonide heterostructure devices at SCD (Invited Paper) [9370-60]

$937022 \quad 10 \mu \mathrm{m}$ pitch design of HgCdTe diode array in Sofradir [9370-62] 
937023 Extended-shortwave infrared unipolar barrier detectors (Invited Paper) [9370-63]

937024 Military applications for high-performance thermal imaging (Invited Paper) [9370-64]

NONLINEAR OPTICS

937025 High-power nonlinear frequency converted laser diodes (Invited Paper) [9370-65]

937026 High-power tunable two-color VECSEL for on-demand wavelength generation (Invited Paper) [9370-66]

937028 Integrated optical sensing technologies on Si (Invited Paper) [9370-68]

INTERBAND CASCADE LASERS

937029 Interband cascade lasers with high CW power and brightness (Invited Paper) [9370-69]

9370 2A Widely tunable interband cascade lasers for the mid-infrared (Invited Paper) [9370-70]

9370 2B Interband cascade laser based absorption sensor for ppb-level formaldehyde detection [9370-71]

\section{LASER DIODES}

9370 2C Review of Al-free active region laser diodes on GaAs for pumping applications (Invited Paper) [9370-72]

9370 2D High-power ultrafast and broadly tunable quantum-dot lasers (Invited Paper) [9370-73]

$93702 \mathrm{E} \quad$ High-performance GaSb laser diodes and diode arrays in the 2.1-3.3 micron wavelength range for sensing and defense applications [9370-74]

\section{GAN CHALLENGES}

$93702 \mathrm{~F} \quad$ Polarization-free GaN emitters in the ultraviolet and visible spectra via heterointegration on CMOS-compatible Si (100) (Invited Paper) [9370-75]

\section{QUANTUM OPTICS}

$93702 R \quad$ Orbital angular momentum injection in a polariton superfluid (Invited Paper) [9370-80]

$93702 \mathrm{~T} \quad$ Exciton-polariton Bose-Einstein condensation with a polymer at room temperature (Invited Paper) [9370-82]

$93702 \mathrm{U}$ Manipulation of electrical flicker-noise and line narrowing in free-running quantumcascade lasers (Invited Paper) [9370-1111] 
RECENT ADVANCES IN QUANTUM SENSING AND NANOPHOTONICS III

9370 2W Monolithic coupling of QCLs in evanescent waveguides on InP (Invited Paper) [9370-84]

$93702 \mathrm{X} \quad$ Low-dimensional II-VI oxide-based semiconductor nanostructure photodetectors for light sensing (Invited Paper) [9370-98]

$93702 Y \quad$ Monolithic optical frequency comb based on quantum dashed mode locked lasers for $\mathrm{Tb} / \mathrm{s}$ data transmission (Invited Paper) [9370-85]

$93702 Z$ Tuning of superconducting nanowire single-photon detector parameters for VLSI circuit testing using time-resolved emission [9370-86]

937030 Introducing Fourier-domain mobility spectrum analysis (FMSA) to deduce multi-component carrier mobility and density [9370-110]

POSTER SESSION

937032 Long-wavelength interband cascade infrared photodetectors operating above room temperature [9370-93]

937033 All-optical reservoir computing system based on InGaAsP ring resonators for high-speed identification and optical routing in optical networks [9370-94]

937034 Multifunctional diffractive optical elements for the generation of higher order Bessel-like beams [9370-97]

$937036 \quad$ Nanomaterials coated multiplexed fiber Bragg grating for multiparameter sensing [9370-100]

937038 Electrical isolation of Type II InAs/InGaSb superlattices from GaSb substrates [9370-102] 


\title{
Authors
}

Numbers in the index correspond to the last two digits of the six-digit citation identifier (CID) article numbering system used in Proceedings of SPIE. The first four digits reflect the volume number. Base 36 numbering is employed for the last two digits and indicates the order of articles within the volume. Numbers start with 00, 01, 02, 03, 04, 05, 06, 07, 08, 09, OA, OB...0Z, followed by 10-12, 20-2Z, etc.

\author{
Abautret, J., ON \\ Abell, J., 29 \\ Abgrall, Michel, 11 \\ Abolghasem, P., 28 \\ Accard, A., 2Y \\ Achard, Jocelyn, $1 \mathrm{~V}$ \\ Acher, Olivier, 07 \\ Albert, S., 17 \\ Allen, Jeffery W., 19 \\ Allen, Monica S., 19 \\ Allred, James J., OV \\ Amy-Klein, Anne, 11 \\ Andersen, Peter E., 25 \\ Appavoo, Kannatassen, $1 \mathrm{C}$ \\ Argall, Brenna D., IY \\ Argence, Bérengère, 11 \\ Asokan, S., 36 \\ Aytac, Y., OJ \\ Babcock, S. E., OE \\ Bahgat Shehata, A., $2 Z$ \\ Bajek, D., 2D \\ Bardou, N., 18 \\ Barry, L. P., $2 Y$ \\ Bartalini, S., 1D, IF \\ Bayram, C., 2F \\ Bébé Manga Lobé, J., 2C \\ Beck, Mattias, OD, $1 \mathrm{E}$ \\ Beere, H. E., OA \\ Bengoechea-Encabo, A., 17 \\ Benisty, Henri, 04 \\ Berney, R., 38 \\ Berthoz, Jocelyn, 00, 22 \\ Bewley, W. W., 29 \\ Bhattacharya, Shanti, 34 \\ Blaser, Stephane, 11 \\ Boggess, T. F., OJ \\ Bogris, A., OT \\ Borri, Simone, OY \\ Boskovic, D., 12 \\ Boso, Gianluca, 1S, IT \\ Botez, D., OE \\ Bouchon, Patrick, $1 \mathrm{P}$ \\ Boulier, T., 2R \\ Boutami, Salim, 04 \\ Bramati, A., 1M, 2R \\ Brambilla, Massimo, 13, 15 \\ Brener, Igal, 05 \\ Bronzi, Danilo, $1 \mathrm{U}$ \\ Brown, Dean P., 19
}

Brown, G. J., OH, 38

Browning, C., $2 Y$

Buelow, P., OE

Buttafava, Mauro, $1 T$

Calandri, Niccolò, $1 T$

Calleja, E., 17

Calligaro, M., 2C

Calò, C., $2 Y$

Campa, A., ID

Cancellieri, E., 2R

Canedy, C. L., 29

Cao, Yingchun, OV, OW, 2B

Cappelli, Francesco, 11

Carbone, L., IM

Carbonnelle, M., 2C

Carletti, L., $1 \mathrm{~J}$

Carpenter, Lewis G., $1 \mathrm{~K}$

Carras, M., OD, 14, 2W

Carrère, Emmanuel, 22

Cataluna, M. A., 2D

Cayron, C., 2C

Chanteau, Bruno, 11

Chardonnet, Christian, 11

Cheng, C. W., 2F

Chenrayan, G. Aravind, 36

Chevalier, Paul, IP

Chipaux, Mayeul, IV

Choi, Young Wan, 1B

Christol, P., ON, $1 Z$

Chung, U-Chan, 05

Columbo, Lorenzo L., 13, 15

Consolino, L., 1D, IF

Contini, Davide, $1 \mathrm{U}$

Coolen, L., IM

Cooper, Peter A., 1K

Cordat, A., ON

Costantini, Daniele, 04

Coutrot, Anne-Lise, 04

Cui, Boya, 30

Dabbicco, Maurizio, 13, 15

Dalla Mora, Alberto, $1 \mathrm{U}$

D'Amato, Francesco, OY, IF

Dargent, Loïc, 22

Darquié, Benoît, 11

Daussy, Christophe, 11

De Angelis, Francesco, 1A

de Mierry, P., 17

Debnath, M. C., 23

Debuisschert, Thierry, IV 
Deev, Andrei, 10

Degl'Innocenti, R., OA

Delmas, M., $1 \mathrm{Z}$

De Mierry, P. 17

De Natale, Paolo, OY, ID, IF

Derelle, S., 1 Z

Di Falco, A., OR

Ding, Y., 2D

Dipalo, Michele, 1A

Domenic, Filip, 05

Drisse, $0 ., 2 \mathrm{C}$

Du, X., 23

Dubertret, B., $1 \mathrm{M}$

Ducournau, Guillaume, 08

Dupuis, C., 18

Dvinelis, Edgaras, 2E

Earles, T., OE

Ekawa, Mitsuru, OF

Elhamri, S., OH, 38

Elissalde, Cathy, 05

Evirgen, A., ON

Faist, Jérôme, OD, 1E, 11

Fallahi, Mahmoud, 26

Fastenau, Joel M., 32

Feezell, Daniel, 19

Ferré, S., OD, 14

Ferron, Alexandre, 00

Fice, Martyn J., OB

Fischer, M., 2A

Flatté, Michael E., OJ, OK

Fratalocchi, A., OR

Fuchs, F., 12

Garcia, M., 2C

Gates, James C., $1 \mathrm{~K}$

Gawith, Corin B. E., 1K

Geiser, Markus, 11

Giacobino, E., 2R

Giard, E., 12

Gilles, C., 2W

Glorieux, Q., 2R

Golding, T. D., 23

Graham, Chris, OB

Grahmann, J., 12

Gravrand, Olivier, 00

Grayson, M., OL, 30

Grazulis, L., $\mathrm{OH}$

Greffet, Jean-Jacques, 04, IP

Greibus, Mindaugas, 2E

Grein, Christoph H., OK

Griffin, Robert J., OV, OW

Grille, Romain, 00

Grillet, C., $1 \mathrm{~J}$

Grillot, F., 14

Gunapala, Sarath D., OM

Haglund, Richard F. Jr., $1 \mathrm{C}$

Haïdar, Riad, 18, 1P

Hansen, Anders K., 25

Hashimoto, Jun-ichi, OF

Haugan, H. J., OH, 38

Hawkins, S. D., OJ
Helmy, Amr S., 28

Hessenius, Chris, 26

Hill, Cory J., OM

Hirohata, Toru, $2 \mathrm{U}$

Höfling, S., 2A

Hoglund, Linda, OM

Holmes, Christopher, 1K

Hugger, S., 12

Hugi, Andreas, 11

Hugonin, Jean-Paul, 04

Imbert, J., $1 \mathrm{Z}$

Jacassi, Andrea, IA

Jacques, Vincent, $1 \mathrm{~V}$

Jaeck, J., 18

Jain, M., 23

Jamois, C., $1 \mathrm{~J}$

Jarvis, J.-P., 12

Javaux, C., 1M

Jensen, Ole Bjarlin, 25

Jessop, D. S., OA

Jiang, Dongfang, OV, OW

Jiang, Wenzhe, OV, OW, 2B

Johnson, Matthew B., 32

Jumpertz, L., 14

Junesand, C., OD

Jung, Byung Gue, 1B

Kamp, M., 2A

Kang, D., 28

Kapsalis, Alexandros, OT, 33

Katsuyama, Tsukuru, OF

Kaušylas, Mindaugas, 2E

Kazemi, Alireza, 19

Keay, Joel C., 32

$\mathrm{Keo}$, Sam A., OM

Kerlain, Alexandre, 00, 22

Ki, Hyun Chul, 1B

Kim, C. S., 29

Kim, Doo Gun, 1B

Kim, Hong-Seung, 1B

Kim, Hwe Jong, 1B

Kim, J. K., OJ

Kim, J., 2F

Kim, Jun Oh, 19

Kim, M., 29

Kim, Seon Hoon, 1B

Kim, T. W., OE

Kim, Tae Un, 1B

Kirch, J. D., OE

Klem, J. F., OJ

Klipstein, Philip, 20

Ko, Yeong Hwan, $2 X$

Koeth, J., 2A

Kong, X., 17

Korzh, Boris, is

Krakowski, M., 2C, 2D

Krauss, T. F., OR

Kriesel, Jason M., OZ, 16

Krishna, Sanjay, 19

Kuech, T. F., OE

Kužel, Petr, 05 
Lampin, Jean-François, 08

Lamponi, M., 2C

Larrue, A., 2C

Latzel, Philipp, 08

Laverdant, J., $1 \mathrm{M}$

Leclerc, J.-L., $1 \mathrm{~J}$

Lecomte, M., 2C

Le Coq, Yann, 11

Lefebvre, Anthony, 04

Lei, Lin, 32

Lelarge, F., $2 Y$

Lemaître, A., 18

Letartre, X., $1 \mathrm{~J}$

Lethiec, C., $1 \mathrm{M}$

Lewicki, Rafal, OW

Li, Lu, 32

Ligeret, $\mathrm{V}$., $2 \mathrm{C}$

Lin, Z., $1 \mathrm{~J}$

Liu, Amy W. K., 32

Liv, C., OR

Liverini, $\mathrm{V}$., OD

Locatelli, M., IF

Logan, D., 28

Lopez, Olivier, 11

Lopez-Romero, D., 17

Lotfi, Hossein, 32

Lourdudoss, S., OD

Lubyshev, Dmitri, 32

Lueck, M. R., $1 \mathrm{~L}$

Lukowski, Michal, 26

Lunghi, Tommaso, $1 \mathrm{~S}$

Luo, Longqiang, 2B

Lussana, Rudi, IU

Lynch, Stephen G., IK

Maglione, Mario, 05

Mahalingam, K., $\mathrm{OH}$

Mahrt, Rainer F., 2T

Mai, Lijian, 2T

Maillard, Magalie, 00

Maisons, G., 2W

Maître, A., $1 M$

Malerba, Mario, IA

Malta, D., $1 \mathrm{~L}$

Manavaimaran, B., OD

Mansuripur, Masud, OU

Marquier, Francois, 04

Marti, Dominik, 25

Martinez, A., $2 Y$

Masini, L., OA

Masterjohn, C. M., IL

Mawst, L. J., OE

Mazzotti, D., ID

McEwan, Ken, 24

Meijer, Jan, IV

Mennea, Paolo L., $1 \mathrm{~K}$

Merghem, K., $2 Y$

Merritt, C. D., 29

Merten, A., 12

Mesaritakis, Charis, OT, 33

Messina, Gabriele C., 1 A
Metaferia, W., OD

Meyer, J. R., 29

Mezzapesa, Francesco P., 13, 15

Michel, N., 2C

Mishima, T. D., 23

Misra, Abha, 36

Mitchel, W. C., 38

Mitchell, Bill, 19

Mitrofanov, Oleg, 05

Moldovan-Doyen, loana, 04

Monat, C., $1 \mathrm{~J}$

Mori, Hiroki, OF

Mostallino, Roberto, 2C

Mou, Shin, 38

Mounaix, Patrick, 05

Murata, Makoto, OF

Muzilla, M. S., $1 \mathrm{~L}$

Nagaraju, Goli, 2X

Nähle, L., 2A

Nami, Mohsen, 19

Natrella, Michele, OB

Nedelcu, A., ON

Nicolodi, Daniele, 11

Olson, B. V., OJ

Orobtchouk, R., $1 \mathrm{~J}$

Ostendorf, R., 12

Ott, J., 2F

Panapakkam, V., $2 Y$

Pardo, Fabrice, 18, IP

Parillaud, O., 2C

Patimisco, Pietro, OX, OY, 16

Pavanello, Fabio, 08

Pelouard, Jean-Luc, 18, IP

Pennetta, Riccardo, OX, OY

Péré-Laperne, Nicolas, 00, 18, 22

Peretti, R., OD

Perez, J.-P., ON

Petersen, Paul Michael, 25

Peytavit, Emilien, 08

Pezzagna, Sébastien, IV

Phillips, Mark C., $0 Z$

Pisanello, F., 1M

Pitanti, A., OA

Plumhof, Johannes D., 2T

Ponnampalam, Lalitha, $\mathrm{OB}$

Portier, B., 18

Posner, Matthew T., $1 \mathrm{~K}$

Prasad, Vishnu, 36

Pugliese, E., IF

Qiu, Yueming, 32

Rajeev, A., $\mathrm{OE}$

Ramanan, J., 28

Ramdane, A., $2 Y$

Ravaro, M., 1D, 1F

Razeghi, M., $2 F$

Ren, Wei, OV, OW, 2B

Ren, Y., OA

Renaud, Cyril C., OB

Reno, John L., 05

Ribet-Mohamed, I., 1 Z 
Ritchie, D. A., OA

Robert, Y., 2C

Roch, Jean-François, $1 \mathrm{~V}$

Rodriguez, J. B., 1 Z

Rojo-Romeo, P., $1 \mathrm{~J}$

Ronzani, A., OA

Rösch, Markus, $1 \mathrm{E}$

Rosencher, E., 18

Rubaldo, Laurent, 00, 22

Ruggeri, Alessandro, $1 \mathrm{~T}$

Sadana, D. K., 2F

Sampaolo, Angelo, OX, OY, 16

Sanchez, Nancy P., OV, OW

Sanchez-Garcia, M., 17

Sanguinetti, Bruno, 15

Santos, M. B., 23

Sanzaro, Mirko, $1 \mathrm{~T}$

Savich, G. R., 23

Scalari, Giacomo, 1E

Scamarcio, Gaetano, OX, OY, 13, 15, 16

Scarcella, Carmelo, $1 \mathrm{~T}$

Schenk, H., 12

Scherf, Ullrich, 2T

Schevermann, J., 2A

Schires, K., 14

Schulte, K., OE

Seeds, Alwyn J., OB

Shah, Y. D., OA

Shaner, E. A., OJ

Shiu, K. T., 2F

Shivananju, B. N., 36

Siciliani de Cumis, Mario, OY, IF

Sidor, D. E., 23

Šimonytè, leva, 2E

Simozrag, B., OD, 2W

Skokan, M. R., $1 \mathrm{~L}$

Skovgaard, Peter M. W., 25

Smith, Peter G. R., IK

Soibel, Alexander, OM

Songaila, Ramūnas, 2E

Spagnolo, Vincenzo, OX, OY, 16

Stellari, F., $2 Z$

Steveler, E., 18

Stöferle, Thilo, 2T

Syvridis, Dimitris, OT, 33

Taalat, Rachid, 00, 22

Tallaire, Alexandre, IV

Tanahashi, Toshiyuki, OF

Tang, Yang, OL, 30

Taubman, Matthew S., OZ

Temple, D. S., $1 \mathrm{~L}$

Ting, David Z.-Y., OM

Tisa, Simone, $1 \mathrm{U}$

Tittel, Frank K., OV, OW, OX, 2B

Tosi, Alberto, 1T, $1 \mathrm{U}$

Trampert, A., 17

Tran, M., 2C

Tredicucci, A., OA

Trinité, V., $1 \mathrm{Z}$

Trinkūnas, Augustinas, 2E
Tsuji, Yukihiro, OF

Urbas, Augustine, 19

Vallon, H., 1M

Varma, Manoj M., 36

Vest, B., 18

Viciani, Silvia, OY

Vick, E. P., $1 \mathrm{~L}$

Vijayakumar, A., 34

Viktorovitch, P., $1 \mathrm{~J}$

Villa, Federica, $1 \mathrm{U}$

Villares, Gustavo, 11

Vinet, E., 2C

Vitiello, Miriam S., OY, 1D, 1F

Vizbaras, Augustinas, 2E

Vizbaras, Kristijonas, 2E

von Bandel, N., 2C

von Edlinger, M., 2A

Vujicic, V., $2 Y$

Vurgaftman, I., 29

Wagner, J., 12

Wallart, Xavier, 08

Watts, R. T., 2Y

Weih, R., 2A

Weiss, Sharon M., $1 C$

Wenner, Brett R., 19

White, S. E., 2D

Wicks, G. W., 23

Wood, A., OE

Wu, Sheng, 10

Xavier, Stéphane, IV

Yamanishi, Masamichi, 2U

Yang, Rui Q., 32

Yoshinaga, Hiroyuki, OF

Yu, Jae Su, $2 X$

Zaknoune, Mohammed, 08

Zappa, Franco, $1 \mathrm{U}$

Zbinden, Hugo, $1 \mathrm{~S}$

Zhu, Y., $2 \mathrm{~F}$

Žukauskas, Tomas, 2E

Zúñiga-Pérez, J., 17

Zutter, B., OE 


\title{
Conference Committee
}

\author{
Symposium Chairs
}

David L. Andrews, University of East Anglia (United Kingdom)

Alexei L. Glebov, OptiGrate Corporation (United States)

Symposium Co-chairs

Jean-Emmanuel Broquin, IMEP-LAHC (France)

Shibin Jiang, AdValue Photonics, Inc. (United States)

Program Track Chair

Ali Adibi, Georgia Institute of Technology (United States)

Conference Chair

Manijeh Razeghi, Northwestern University (United States)

Conference Co-chairs

Eric Tournié, Université Montpellier 2 (France)

Gail J. Brown, Air Force Research Laboratory (United States)

Conference Program Committee

Jong Hyeob Baek, Korea Photonics Technology Institute

(Korea, Republic of)

Can Bayram, University of Illinois at Urbana-Champaign (United States)

David A. Cardimona, Air Force Research Laboratory (United States)

Jérôme Faist, ETH Zürich (Switzerland)

Siamak Forouhar, Jet Propulsion Laboratory (United States)

Michael D. Gerhold, U.S. Army Research Office (United States)

Frédéric Grillot, Télécom ParisTech (France)

Yasar Gurbuz, Sabanci University (Turkey)

Sven Höfling, University of St. Andrews (United Kingdom)

Jean-Pierre Huignard, Jphopto (France)

Woo-Gwang Jung, Kookmin University (Korea, Republic of)

Tsukuru Katsuyama, Sumitomo Electric Industries, Ltd. (Japan)

Jean F. Kelly, Pacific Northwest National Laboratory (United States)

Michel Krakowski, Thales Research \& Technology (France)

Kwok Keung Law, Naval Air Warfare Center Weapons Division

(United States) 
Giuseppe Leo, Université Paris 7-Denis Diderot (France)

Amy W. K. Liu, IQE Inc. (United States)

Jerry R. Meyer, U.S. Naval Research Laboratory (United States)

Maya Mikhaliova, loffe Physical-Technical Institute

(Russian Federation)

Jan Misiewicz, Wroclaw University of Technology (Poland)

Oleg Mitrofanov, University College London (United Kingdom)

Ekmel Özbay, Bilkent Üniversity (Turkey)

Dimitris Pavlidis, Boston University (United States)

Mark C. Phillips, Pacific Northwest National Laboratory (United States)

Divyang Shah, National Reconnaissance Office (United States)

Carlo Sirtori, Université Paris 7-Denis Diderot (France)

Marija Strojnik Scholl, Center de Investigaciones en Óptica, A.C. (Mexico)

Meimei Tidrow, U.S. Army Night Vision \& Electronic Sensors Directorate (United States)

Alberto Tosi, Politecnico di Milano (Italy)

Alessandro Tredicucci, Laboratorio NEST (Italy)

Sheng Wu, California Institute of Technology (United States)

Rui Q. Yang, The University of Oklahoma (United States)

John M. Zavada, National Science Foundation (United States)

Session Chairs

1 Keynote Session

Gail J. Brown, Air Force Research Laboratory (United States)

2 IR Emitters and Near-field Characterization

Meimei Tidrow, U.S. Army Night Vision \& Electronic Sensors Directorate (United States)

Andrea Fiore, Technische Universiteit Eindhoven (Netherlands)

$3 \mathrm{THz}$ Sources

Pascale Senellart, Laboratoire de Photonique et de Nanostructures

(France)

Miriam S. Vitiello, Consiglio Nazionale delle Ricerche (Italy)

4 Mid-IR QCL Structures

Jerry R. Meyer, U.S. Naval Research Laboratory (United States)

Christian Grillet, Ecole Centrale de Lyon (France)

5 IR Photodetector Materials

Martin Kamp, Julius-Maximilians-Univ. Würzburg (Germany)

Can Bayram, University of Illinois at Urbana-Champaign

(United States) 
Sb-based IR Photodetectors

Philippe Christol, Institut d'Electronique du Sud (France)

Gary W. Wicks, University of Rochester (United States)

7 Optomechanics/Micro-Resonators

Giuseppe Leo, Université Paris 7-Denis Diderot (France)

Quentin C. Glorieux, National Institute of Standards and Technology (United States)

$8 \quad$ IR Sensing

Joachim Wagner, Fraunhofer-Institut für Angewandte Festkörperphysik (Germany)

Francesco De Angelis, Istituto Italiano di Tecnologia (Italy)

9 QCLs and Applications I

Paolo De Natale, Istituto Nazionale di Ottica (Italy)

Paul M. Petersen, Technical University of Denmark (Denmark)

10 Keynote Session

Manijeh Razeghi, Northwestern University (United States)

11 Plasmonic Detection and Sensing

Frank K. Tittel, Rice University (United States)

Vincenzo Spagnolo, Politecnico di Bari (Italy)

12 QCLs and Applications II

Ivan Favero, Université Paris 7-Denis Diderot (France)

Maria Ana Cataluna, University of Dundee (United Kingdom)

13 Integration Technologies

Lorenzo L. Columbo, CNR-Istituto di Fotonica e Nanotecnologie (Italy)

Sebastian Lourdudoss, KTH Royal Institute of Technology (Sweden)

14 Recent Advances in Quantum Sensing and Nanophotonics I

Manijeh Razeghi, Northwestern University (United States)

Michel Krakowski, Thales Research \& Technology (France)

15 Recent Advances in Quantum Sensing and Nanophotonics II

Manijeh Razeghi, Northwestern University (United States)

Michel Krakowski, Thales Research \& Technology (France)

16 Keynote Session

Manijeh Razeghi, Northwestern University (United States) 
17 IR Photodetectors

Alexander Soibel, Jet Propulsion Laboratory (United States)

Gail J. Brown, Air Force Research Laboratory (United States)

18 Nonlinear Optics

Juliette Mangeney, Ecole Normale Supérieure (France)

Tobias J. Kippenberg, Ecole Polytechnique Fédérale de Lausanne

(Switzerland)

19 Interband Cascade Lasers

Stefano Barbieri, Université Paris 7-Denis Diderot (France)

Riccardo Degl'Innocenti, University of Cambridge (United Kingdom)

20 Laser Diodes

Jean-Pierre Huignard, Jphopto (France)

Anne Amy-Klein, Laboratoire de Physique des Lasers (France)

21 GaN Challenges

Enrique Calleja, Universidad Politécnica de Madrid (Spain)

Luke J. Mawst, University of Wisconsin-Madison (United States)

22 Late-Breaking Results and Awards

Manijeh Razeghi, Northwestern University (United States)

23 Keynote Session

Manijeh Razeghi, Northwestern University (United States)

24 Quantum Optics

Carlo Sirtori, Université Paris 7-Denis Diderot (France)

Andrea Di Falco, University of St. Andrews (United Kingdom)

25 Recent Advances in Quantum Sensing and Nanophotonics III

Manijeh Razeghi, Northwestern University (United States)

Michel Krakowski, Thales Research \& Technology (France) 